\title{
La SIFEM : un redéploiement prometteur
}

À la fin de l'année 2016, le conseil d'administration de la Société internationale francophone d'éducation médicale (SIFEM) a décidé de réaffirmer clairement les missions de l'organisation, en lien avec son projet fondateur qui vise à en faire la société savante francophone de référence, capable d'attirer, grâce à son dynamisme et sa perspective fédératrice, toutes les personnes concernées par la formation des différents professionnels de la santé.

Une telle ambition implique qu'elle soit en mesure d'offrir à ses membres toutes les ressources nécessaires pour rendre plus intelligibles les différents problèmes auxquels ils sont confrontés dans le cadre de leurs multiples pratiques professionnelles, afin d'améliorer la pertinence et la cohérence de celles-ci, au bénéfice, in fine, de la qualité et de l'efficience des soins aux patients. Il s'agit, par exemple: de favoriser le recensement, l'analyse et l'évaluation de dispositifs de formation innovants; de créer des conditions permettant l'explicitation, la mutualisation et la diffusion de savoirs expérientiels; d'aider les promoteurs d'outils didactiques ou de formats pédagogiques à en examiner les enjeux et à en documenter les retombées; de rendre disponibles et abordables, grâce à une médiatisation raisonnablement pragmatique, mais critique et non réductrice, les cadres théoriques et conceptuels qui permettent de valoriser les uns et les autres, en permettant d'identifier les conditions de leur transfert et d'accroître ainsi leur portée générale.

En somme, dans la droite ligne de la perspective du Scholarship of Teaching and Learning [1], il s'agit d'aider les différents acteurs du champ de la formation des professionnels de la santé à appréhender de façon méthodique, dans un continuum, les différentes dimensions de leurs activités et de leurs pratiques de formation, notamment politiques, sociales, professionnelles, savantes et scientifiques, au regard desquelles, selon les différentes situations, ils s'efforcent de bien enseigner, d'enseigner de façon savante ou de contribuer au développement du savoir sur l'enseignement et l'apprentissage dans leur champ. Cet éditorial vise à faire le point quant aux moyens mis en œuvre pour atteindre ces objectifs.

L'une des premières préoccupations en lien avec ce nouveau mandat pour la SIFEM a dès lors consisté à mettre à la disposition de ses membres divers outils facilitant les échanges, ou différentes ressources techno- logiques, propres à favoriser, par exemple, les travaux des groupes travaillant sur des thématiques communes. Notre site web $^{1}$ est ainsi conçu comme un véritable portail, qui propose de nombreuses rubriques en lien avec la dimension pluriprofessionnelle de la société. Le site est très régulièrement mis à jour et on y trouve en particulier les résultats d'une veille éditoriale, scientifique et documentaire, concernant les actualités dans le domaine des formations en santé. Nous vous invitons à l'explorer pour vous tenir au courant de la vie de la société et à vous abonner à ses comptes sociaux. Vous y trouverez notamment les annonces des congrès et ateliers à venir, ainsi que des liens vers différentes institutions et sociétés savantes, ou encore des ressources utiles pour vos pratiques de formation et de recherche.

La tenue d'un grand congrès francophone annuel en éducation des sciences de la santé devient une réalité. Nous avons établi à cet effet un partenariat avec les organisateurs du Forum international francophone de pédagogie des sciences de la santé (Canada), afin d'organiser en alternance, un an sur deux, un congrès d'éducation des sciences de la santé, respectivement au Québec et dans le reste de la francophonie. Cette alternance débutera avec le $8^{\mathrm{e}}$ Forum, qui aura lieu à Montréal du 29 au 31 mai 2019, sur le thème «Évaluer pour apprendre ${ }^{2}$. Elle se poursuivra par le congrès de la SIFEM qui se tiendra à Strasbourg, du 13 au 15 mai 2020.

Par ailleurs, la SIFEM a inauguré cette année un nouveau dispositif de formation thématique approfondie: les «Ateliers d'automne de la SIFEM ». L'édition 2018 s'est tenue à Brest au mois de septembre. Trois thèmes ont été explorés pendant quatre demi-journées : ingénierie de la professionnalisation et éthique des curriculums, mesure et évaluation et patient-partenaire de soins. Les participants aux ateliers ont exprimé un niveau très élevé de satisfaction et ils ont fortement encouragé les responsables de la société à en faire un évènement pérenne. C'est ainsi que, dès l'an prochain, la deuxième édition des Ateliers d'automne de la SIFEM se tiendra à Nice, du 16 au 18 octobre 2019. Pour en savoir davantage, nous vous invitons à visiter régulièrement la page ${ }^{3}$.

\footnotetext{
${ }^{1}$ https://sifem.net/fr/accueil.

2 https://www.fpedago.org/fr.

${ }^{3}$ https://sifem.net/fr/ateliers-automne2018.
} 
Enfin, sous l'égide de la SIFEM, plusieurs groupes thématiques se sont constitués et échangent régulièrement afin d'offrir à terme différentes ressources ou services aux membres de la société, par exemple, sous la forme de contributions éditoriales et notamment d'articles didactiques. Citons, par exemple, les groupes «Mesure et évaluation», «Éthique des curriculums de formation en santé», «Le numérique en pédagogie » et «Raisonnement clinique». Nous vous invitons à découvrir les activités de ces groupes et à vous joindre à eux, en visitant la page dédiée ${ }^{4}$.

La revue Pédagogie Médicale, organe officiel d'expression de la SIFEM, constitue une ressource extraordinaire pour la communauté francophone en éducation des sciences de la santé. Créée en 2000, et publiée de façon trimestrielle, elle est un organe privilégié de communication, d'échanges et de débats, de partage d'expériences et de diffusion des résultats des travaux de recherche dans toutes les disciplines de la santé. Depuis un an, elle est publiée exclusivement au format électronique ${ }^{5}$, ce qui en facilite la diffusion. Désormais, dès qu'un nouveau numéro est publié, tous les membres de la SIFEM reçoivent automatiquement par courriel une notification. Quelques contraintes conjoncturelles ont entraîné une surcharge passagère du comité de rédaction. Ceci a conduit à renforcer celui-ci, Nicolas Fernandez épaulant désormais Jean Jouquan en tant que rédacteur en chef adjoint. Cette opportunité a aussi été saisie pour renforcer la dimension pluriprofessionnelle du comité de rédaction. Traduisant le dynamisme des membres de la société et, au-delà, de toutes les personnes qui reconnaissent en elle le moyen privilégié de valoriser leurs travaux, le nombre de manuscrits soumis ne faiblit pas (environ une soixantaine par an). Le comité de rédaction s'efforce d'exploiter judicieusement cette richesse, en considérant que le processus d'évaluation éditoriale constitue, en tant que tel, une opportunité privilégiée de formation contextualisée à la recherche et à la publication, qui fait partie intégrante des missions de la société. Une telle préoccupation est exigeante et sollicite de nombreuses ressources. Les conditions sont à nouveau réunies pour en préserver la réactivité et le rythme régulier des publications, incluant les numéros en retard, devrait être restauré au cours de l'année 2019. N'hésitez donc pas à partager vos résultats de recherche et vos idées innovantes sous la forme de manuscrits. Le site de la revue Pédagogie Médicale, à partir duquel il est possible de consulter et télécharger les articles des numéros archivés, est accessible directement à partir du portail de la SIFEM $^{6}$.
L'approbation éthique préalable des projets de recherche est de plus en plus exigée par les comités de rédaction des revues internationales lors de la soumission d'articles. Elle constitue même un critère obligatoire pour l'indexation des périodiques dans les grandes bases de données scientifiques internationales. La recherche en éducation des professions de la santé est beaucoup plus proche, sur les plans méthodologique et épistémologique, de la recherche en sciences humaines et sociales que de la recherche en sciences bio-cliniques. Lorsqu'ils sont sollicités, les comités d'éthique pour la recherche clinique et biomédicale se déclarent ainsi souvent incompétents pour examiner le caractère éthiquement acceptable de tels projets. Pour pallier de telles difficultés et répondre aux besoins de ses membres, la SIFEM a donc créé un Comité pour l'intégrité et l'éthique de la recherche en éducation des professions de la santé. La soumission des projets se fait sur la page concernée ${ }^{7}$.

La SIFEM compte à présent plus de 400 membres. Sa croissance rapide témoigne de la vitalité de la société. Le nombre important de participants aux ateliers et aux congrès démontre l'existence d'un réel sentiment d'appartenance à une communauté, autour des valeurs partagées que sont l'innovation, la collaboration interprofessionnelle, la rigueur et l'équité, constitutives d'une éthique institutionnelle. Chacun pourra constater que notre société savante se positionne désormais au cœur des échanges et des débats dans le champ de la formation des professionnels de la santé.

$$
\begin{array}{r}
\text { Bernard CHARLIN } \\
\text { Président de la SIFEM } \\
\text { Anne DEMEESTER } \\
\text { Thiere-présidente et trésorière de la SIFEM } \\
\text { Thierry PELCCIA } \\
\text { Secrétaire général de la SIFEM } \\
\text { Jean JOUQUAN } \\
\text { Rédacteur en chef de Pédagogie Médicale } \\
\text { Nicolas FERNANDEZ } \\
\text { Rédacteur en chef-adjoint de Pédagogie Médicale }
\end{array}
$$

*Mailto : bernard.charlin@umontreal.ca

\section{Référence}

1. Simpson D, Fincher RM, Hafler JP, Irby DM, Richards BF, Rosenfeld GC, Viggiano TR. Advancing educators and education by defining the components and evidence associated with educational leadership. Med Educ 2007;41:1002-1009.

\footnotetext{
${ }^{4}$ https://sifem.net/fr/groupes-thematiques.

${ }^{5}$ https://www.pedagogie-medicale.org.

${ }^{6}$ https://sifem.net/fr/pedagogie-medicale.
}

$\overline{{ }^{7} \text { https://sifem.net/fr/comite-ethique-recherche. }}$ 